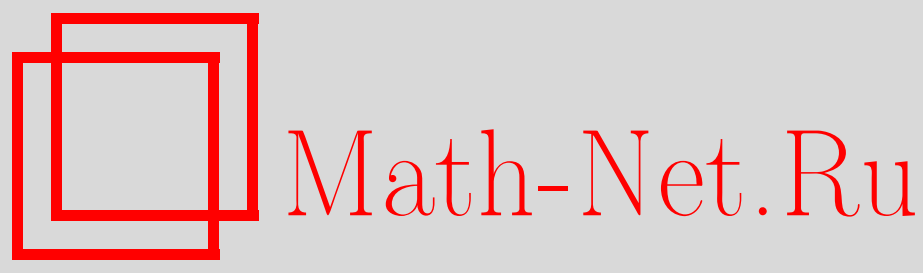

В. Ф. Лось, Нелинейные обобщенные управляющие уравнения и учет начальных корреляций, ТМФ, 2009, том 160, номер 2, 304-330

DOI: https://doi.org/10.4213/tmf6400

Использование Общероссийского математического портала Math-Net.Ru подразумевает, что вы прочитали и согласны с пользовательским соглашением http://www . mathnet.ru/rus/agreement

Параметры загрузки:

IP: 54.164 .48 .24

26 апреля 2023 г., 13:01:28

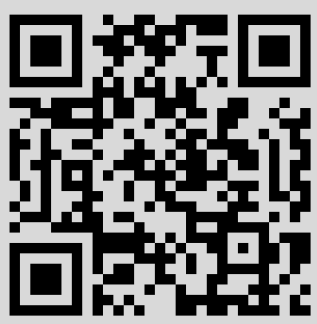




\section{НЕЛИНЕЙНЫЕ ОБОБЩЕННЫЕ УПРАВЛЯЮЩИЕ УРАВНЕНИЯ И УЧЕТ НАЧАЛЬНЫХ КОРРЕЛЯЦИЙ}

Развит новый метод, основанный на использовании зависящего от времени (вообще говоря, не проекционного) оператора, преобразующего функцию распределения (статистический оператор) полной системы к соответствующему (релевантному) виду, позволяющему выводить новые точные нелинейные обобщенные управляющие уравнения (ОУУ). Выведенное неоднородное нелинейное ОУУ является обобщением линейного ОУУ Накаджимы-Цванцига и может рассматриваться как альтернатива цепочки ББГКИ. Оно может быть использовано для получения как нелинейных, так и линейных эволюционных уравнений. Как и в обычном линейном ОУУ, имеется неоднородный член, содержащий все многочастичные начальные корреляции. Чтобы включить в рассмотрение начальные корреляции, полученное неоднородное нелинейное ОУУ преобразовано к однородному виду с помощью предложенного ранее метода. При этом не используются никакие стандартные приближения, такие как приближение случайных фаз или принцип Боголюбова ослабления начальных корреляций. Полученное точное однородное нелинейное ОУУ описывает все стадии эволюции рассматриваемой подсистемы; при этом начальные корреляции рассматриваются единообразно со столкновениями посредством модифицированного ядра памяти. В качестве приложения получено новое однородное нелинейное уравнение, сохраняющее начальные корреляции, для одночастичной функции распределения пространственно неоднородного неидеального газа классических частиц. В отличие от существующих подходов, это уравнение справедливо на всех временны́х масштабах и учитывает влияние парных столкновений и начальных корреляций на диссипативные и недиссипативные характеристики системы в соответствии с принятым приближением (линейным по плотности газа). Показано, что на кинетическом временно́м масштабе обратимые во времени члены, обусловленные начальными корреляциями, обращаются в нуль (если динамика частиц обладает свойством перемешивания), и поэтому данное уравнение можно преобразовать в уравнения Власова-Ландау и Больцмана без каких-либо обычно используемых дополнительных приближений. Таким образом, можно проследить весь процесс перехода от начальной обратимой стадии эволюции к необратимой кинетической стадии.

Ключевые слова: нелинейные управляющие уравнения, начальные корреляции, необратимость.

* Институт магнетизма НАН Украины, Киев, Украина.

E-mail: vlos@imag.kiev.ua, victorlos@mail.ru 
Посвящается памяти академика Н.Н. Боголюбова

\section{1. ВВЕДЕНИЕ}

Вывод уравнений, позволяющих описывать измеримые (статистические средние) значения, характеризующие неравновесное состояние многочастичной системы, остается одной из основных задач статистической физики. Естественно ожидать, что эти уравнения должны, вообще говоря, быть нелинейными эволюционными уравнениями, переходящими в кинетические (или другие необратимые) уравнения на некотором временно́м масштабе. Принципиальный вопрос заключается в том, как строго вывести такие необратимые уравнения из фундаментальных микроскопических (обратимых) классических или квантовых динамических уравнений и что является причиной наблюдаемой необратимости. Для решения этих задач обычно используется несколько подходов, которые, как правило, исходят из линейного уравнения Лиувилля-фон Неймана для функции распределения (статистического оператора) рассматриваемой системы многих тел.

Один из подходов приводит к цепочке связанных дифференциальных уравнений первого порядка для многочастичных функций распределения, известной как иерархия Боголюбова-Борна-Грина-Кирквуда-Ивона (ББГКИ) [1]. Наиболее последовательный подход к расцеплению цепочки ББГКИ был развит Боголюбовым [1]. Он предложил принцип ослабления начальных корреляций, из которого следует, что на достаточно больших временах $t-t_{0} \gg t_{\text {cor }}\left(t_{\text {cor }}-\right.$ время корреляции, определяемое межчастичным взаимодействием) все начальные корреляции (существующие в начальный момент времени $t_{0}$ ) затухают, вследствие чего зависимость многочастичных функций распределения от времени полностью определяется зависимостью от времени одночастичной функции распределения (анзац Боголюбова). При этом предполагается существование временно́го интервала

$$
t_{\text {cor }} \ll t-t_{0} \ll t_{\text {rel }}
$$

где $t_{\mathrm{rel}}$ - время релаксации для одночастичной функции распределения, на котором справедливо приближенное (на данном большом промежутке времени) преобразование неоднородного (включающего двухчастичные корреляции) уравнения для одночастичной функции распределения (относящейся к иерархии ББГКИ) в однородное уравнение. Применяя этот подход, Боголюбов успешно вывел кинетические (марковские) уравнения для одночастичной функции распределения, в частности классическое и квантовое уравнения Больцмана, которые описывают временну́ю эволюцию с характерными временами $t_{\text {rel }}$, но не пригодны для начальной стадии эволюции $t_{0} \leqslant t \leqslant t_{\text {cor }}$.

Принцип Боголюбова ослабления начальных корреляций вводится как граничное (начальное) условие для цепочки ББГКИ. Например, для случая классической физики

$$
\begin{gathered}
\lim _{t-t_{0} \rightarrow \infty} U\left(t, t_{0}\right)\left[F_{N}\left(x_{1}, \ldots, x_{N}, t_{0}\right)-f_{r}\left(x_{1}, \ldots, x_{N}, t_{0}\right)\right]=0, \\
f_{r}\left(x_{1}, \ldots, x_{N}, t\right)=\prod_{i=1}^{N} F_{1}\left(x_{i}, t\right),
\end{gathered}
$$

3 Теоретическая и математическая физика, т. 160, № 2, 2009 г. 
где $F_{N}\left(x_{1}, \ldots, x_{N}, t_{0}\right)-N$-частичная функция распределения при $t=t_{0}, x_{i}=$ $\left(\mathbf{x}_{i}, \mathbf{p}_{i}\right)$ - координата $i$-й частицы в фазовом пространстве, а $U\left(t, t_{0}\right)$ - оператор, задающий эволюцию всех координат $x_{i}$ во времени (см. ниже формулу $\left.(9)\right)$. Первое условие (1) подразумевает, что при $t-t_{0} \gg t_{\text {cor }}$ все корреляции между частицами обращаются в нуль, а второе неравенство в (1) $t-t_{0} \ll t_{\text {rel }}$ позволяет пренебречь (в первом приближении по малой плотности частиц $n$ ) запаздыванием во времени и подставить $U\left(t, t_{0}\right) f_{r}\left(t_{0}\right)$ вместо $U\left(t, t_{0}\right) f_{r}(t)$. Таким образом, расцепление цепочки ББГКИ является нелинейной процедурой (2), в результате которой получается замкнутое нелинейное уравнение (типа уравнения Больцмана) для одночастичной функции распределения $F_{1}\left(x_{i}, t\right)$, а в уравнение Лиувилля-фон Неймана вносятся необратимость и нелинейность.

Следует, однако, отметить, что в действительности на кинетическом временно́м масштабе можно пренебречь только начальными корреляциями малого масштаба, для которых $t_{\text {cor }} \ll t_{\text {rel }}$. При получении интеграла столкновений в кинетическом уравнении с учетом столкновений трех (в двумерном случае) и более частиц (члены второго и более высоких порядков по $n$ ) приходится иметь дело с расходимостями, обусловленными, в частности, крупномасштабными начальными корреляциями, для которых $t_{\text {cor }} \gtrsim t_{\text {rel }}$ (см., например, [2], [3]). Таким образом, корректное разложение интеграла столкновений по степеням $n$ и учет кинетических флуктуаций невозможны, если не учитывать начальные корреляции, для которых $t_{\text {cor }} \gtrsim t_{\text {rel }}$ и $l_{\text {cor }} \gtrsim l$, где $l_{\text {cor }}=\bar{v} t_{\text {cor }}, l=\bar{v} t_{\text {rel }}, \bar{v}-$ средняя скорость частиц, а $l-$ средняя длина свободного пробега [4].

В настоящее время не прекращаются попытки строгого вывода уравнения Больцмана (особенно для квантового случая). Это важно как с концептуальной, так и практической точки зрения. Например, следуя работе [5], где было строго получено классическое уравнение Больцмана для системы твердых сфер при очень низких плотностях и для коротких промежутков времени, авторы работы [6] аналогичным (не строгим) образом получили квантовое уравнение Больцмана, однако они использовали факторизованные начальные условия (отсутствие начальных корреляций или приближение случайных фаз при $t=t_{0}$ ), что представляется сомнительным [7]. С другой стороны, следует отметить, что, насколько известно автору, до сих пор нет способа точного учета начальных корреляций в рамках цепочки ББГКИ.

В рамках другого подхода, приводящего к так называемому обобщенному управляющему уравнению (ОУУ) [8]-[10], $N$-частичная функция распределения (или статистический оператор) разбивается с помощью не зависящих от времени проекционных операторов $P$ и $Q$ на существенную (релевантную) и несущественную (нерелевантную) части:

$$
\begin{aligned}
F_{N}\left(x_{1}, \ldots, x_{N}, t\right) & =f_{\mathrm{r}}\left(x_{1}, \ldots, x_{N}, t\right)+f_{\mathrm{i}}\left(x_{1}, \ldots, x_{N}, t\right), \\
f_{\mathrm{r}}\left(x_{1}, \ldots, x_{N}, t\right) & =P F_{N}\left(x_{1}, \ldots, x_{N}, t\right) \\
f_{\mathrm{i}}\left(x_{1}, \ldots, x_{N}, t\right) & =Q F_{N}\left(x_{1}, \ldots, x_{N}, t\right) \\
P+Q & =1 .
\end{aligned}
$$


Заметим, что релевантная и нерелевантная части зависят от координат и/или импульсов всех $N$ частиц, в отличие от редуцированных функций распределения, таких как $F_{1}\left(x_{i}\right)$. Релевантная часть, которая обычно представляет основной интерес, является, как правило, вакуумной (медленно меняющейся) частью функции распределения, т.е. частью без корреляций (подобно $f_{\mathrm{r}}\left(x_{1}, \ldots, x_{N}, t\right)$ из $\left.(2)\right)$. Применяя проекционные операторы $P$ и $Q$ к уравнению Лиувилля-фон Неймана, получаем ОУУ, которое является точным неоднородным немарковским уравнением для релевантной части функции распределения с источником (нерелевантной частью), где содержатся все многочастичные корреляции в начальный момент времени $t_{0}$. Следует подчеркнуть, что используемые в этом подходе не зависящие от времени линейные проекционные операторы $P$ и $Q$ коммутируют с оператором производной по времени в уравнении Лиувилля-фон Неймана для функции распределения полной системы. Поэтому процедура (3) является линейной и преобразует линейное уравнение Лиувилля-фон Неймана в линейное ОУУ. Чтобы исключить начальные многочастичные корреляции (источник), обычно используют принцип Боголюбова ослабления начальных корреляций или просто факторизацию начальных условий (приближение случайных фаз), в результате чего получается приближенное замкнутое линейное однородное ОУУ для релевантной части функции распределения. Последнее далее используется для получения линейного эволюционного (управляющего) уравнения для представляющей интерес редуцированной функции распределения (например, функции распределения для подсистемы, взаимодействующей с термостатом).

Строго говоря, в рамках формализма линейных не зависящих от времени проекционных операторов невозможно получить нелинейное (аналогичное уравнению Больцмана) управляющее уравнение без дополнительных приближений. Поэтому нелинейное уравнение для матрицы плотности часто просто постулируется в виде управляющего уравнения типа уравнения Линдблада с генераторами, параметрически зависящими от матрицы плотности (см., например, [11]).

Сказанное выше, в частности, означает, что правильная трактовка начальных корреляций является ключевым моментом при получении замкнутых эволюционных уравнений. Более того, начальные корреляции могут быть важны при рассмотрении процессов сверхбыстрой релаксации и немарковских процессов, а также для описания явления декогеренции (см., например, [12]). Для учета начальных корреляций недавно был предложен метод, основанный на обычной технике не зависящих от времени проекционных операторов и позволяющий точно привести линейное неоднородное ОУУ со сверткой по времени [8]-[10] и ОУУ без свертки по времени [13], [14] к однородному виду [15], [16]. Этот подход позволяет получать точные линейные однородные ОУУ со сверткой по времени и без свертки по времени, которые учитывают динамику начальных корреляций посредством модифицированных функций памяти, управляющих эволюцией релевантной части функции распределения многочастичной системы. Эти уравнения описывают эволюцию (модифицированную начальными корреляциями) релевантной части функции распределения 
на всех временны́х масштабах, включая начальный $t-t_{0} \lesssim t_{\text {cor }}$, когда, как ожидается, происходит переход от обратимой эволюции к необратимому кинетическому поведению, обусловленному стохастической неустойчивостью динамики системы.

Мы проанализировали, как работает полученное линейное однородное ОУУ со сверткой по времени на примере пространственно однородного разреженного газа классических [15] и квантовых [16] частиц. Однако чтобы из фактически имеющегося линейного однородного ОУУ со сверткой по времени получить нелинейные уравнения (в частности, уравнения Больцмана), описывающие эволюцию газа классических или квантовых частиц, необходимо использовать дополнительное приближение, в котором в одночастичной функции распределения пренебрегается временнь́м запаздыванием. Это связано с тем, что использовавшийся в работе [15] не зависящий от времени проекционный оператор (например, для классических частиц)

$$
P=\left[\prod_{i=2}^{N} F_{1}\left(x_{i}, t_{0}\right)\right] \frac{1}{V^{N-1}} \int d x_{2} \ldots \int d x_{N},
$$

где $V$ - объем системы, определяет релевантную часть $N$-частичной функции распределения в виде выражения

$$
f_{\mathrm{r}}\left(x_{1}, \ldots, x_{N}, t\right)=\left[\prod_{i=2}^{N} F_{1}\left(x_{i}, t_{0}\right)\right] F_{1}\left(x_{1}, t\right),
$$

линейного по зависящей от времени рассматриваемой одночастичной функции распределения $F_{1}\left(x_{1}, t\right) ; F_{N}$ удовлетворяет условию нормировки

$$
\frac{1}{V^{N}} \int d x_{1} \ldots \int d x_{N} F_{N}\left(x_{1}, \ldots, x_{N}, t\right)=1 .
$$

Этот подход можно считать несамосогласованным. Чтобы вывести из полученного линейного уравнения для $F_{1}\left(x_{1}, t\right)$ нелинейное, функцию распределения $F_{1}\left(x_{i}, t_{0}\right)$ можно аппроксимировать функцией $F_{1}\left(x_{i}, t\right)$, если $t-t_{0} \ll t_{\text {rel }}$. Таким образом, в рамках данного подхода мы не связаны необходимостью выполнения первого неравенства (1) $t_{\text {cor }} \ll t-t_{0}$, однако по-прежнему необходимо, чтобы выполнялась его вторая часть. Следовательно, чтобы добиться самосогласованности, т.е. получить нелинейную симметричную по времени релевантную функцию распределения $f_{\mathrm{r}}\left(x_{1}, \ldots, x_{N}, t\right)(2)$ и отбросить упомянутое ограничение по временно́му масштабу, требуется новый подход.

В настоящей работе мы получим новый класс эволюционных уравнений для релевантной части функции распределения (статистического оператора), пригодных для вывода нелинейного и линейного эволюционных уравнений для представляющих интерес редуцированных функций распределения и справедливых на всех временны́х масштабах. Это точные нелинейные неоднородные и однородные ОУУ, получающиеся из уравнения Лиувилля-фон Неймана с помощью, вообще говоря, нелинейного зависящего от времени оператора $P(t)$, преобразующего многочастичную функцию распределения к соответствующему релевантному виду. Зависящий от времени опеpaтор $P(t)$ в общем случае не является проекционным оператором. Для случая не 
зависящего от времени линейного проекционного оператора $P$ выведенные уравнения редуцируются к обычному линейному ОУУ и полученному недавно нелинейному ОУУ [15]. В отличие от линейных уравнений, полученные нелинейные уравнения являются более общими, а потому и более удобными, в частности для получения уравнений типа уравнения Больцмана и изучения пространственно неоднородного неидеального газа частиц, в котором существенную роль играют пространственная нелокальность и запаздывание по времени (см., например, [4]). Кроме того, начальные корреляции могут быть точно включены в рассмотрение посредством преобразования неоднородного нелинейного ОУУ к однородному виду.

Данная работа построена следующим образом. В разделе 2 выводится точное неоднородное нелинейное ОУУ со сверткой по времени для релевантной части функции распределения (статистического оператора), содержащее релевантную часть (включающую начальные корреляции), которая является проблемой для рассмотрения, как и в случае обычного линейного ОУУ. Полученное уравнение является обобщением линейного ОУУ Накаджимы-Цванцига и может быть альтернативой цепочке ББГКИ. В разделе 3 полученное неоднородное нелинейное ОУУ применяется к пространственно неоднородному неидеальному разреженному газу классических частиц посредством выбора подходящего нелинейного оператора $P(t)$. В случае линейной аппроксимации по малому параметру плотности (учитываются только двухчастичные корреляции - столкновения) из этого неоднородного нелинейного ОУУ получается новое неоднородное нелинейное уравнение для одночастичной функции распределения, содержащее самосогласованный власовский член, интеграл столкновений, учитывающий все пространственные и временны́е нелокальности, и нерелевантную часть (двухчастичную корреляционную функцию в начальный момент времени $t_{0}$ ). Используя приближение случайных фаз при $t=t_{0}$ (или принцип Боголюбова ослабления начальных корреляций при $\left.t_{0} \rightarrow-\infty\right)$, мы получаем уравнение Власова-Ландау и нелинейное уравнение Больцмана (эти уравнения выводятся без использования второго ограничения (1)).

Чтобы включить в рассмотрение начальные корреляции, в разделе 4 мы преобразуем полученное в разделе 2 неоднородное нелинейное ОУУ в однородное нелинейное ОУУ со сверткой по времени с помощью метода, предложенного ранее в работе [15]. Это точное уравнение учитывает как начальные корреляции, так и столкновения посредством модифицированной функции памяти, определяющей эволюцию релевантной части функции распределения (статичтического оператора). В разделе 5 мы применяем выведенное однородное нелинейное ОУУ к той же системе, что и в разделе 3. В линейном приближении по параметру плотности мы получаем новое однородное нелинейное уравнение для одночастичной функции распределения, справедливое на всех временнь́х масштабах (не требуются ограничения типа (1)) и учитывающее влияние динамики начальных корреляций на всех этапах процесса эволюции. В линейном по $n$ приближении эволюция столкновений и начальных корреляций (в ядре памяти) определяется точным двухчастичным пропагатором, и в этом смысле полученное уравнение является замкнутым. Если газовая динамика обладает необходимым свойством эргодического перемешивающего потока в фазовом пространстве, то все начальные корреляции обращаются в нуль на кинетическом 
временно́м масштабе и данное уравнение может перейти в необратимое нелинейное уравнение Больцмана с самосогласованным нелинейным власовским членом. При этом не требуются обычно используемые дополнительные допущения, такие как специальные (факторизованные) начальные условия, или принципы. В разделе 6 суммируются полученные результаты.

\section{2. НЕОДНОРОДНОЕ НЕЛИНЕЙНОЕ ОУУ}

Начнем с уравнения Лиувилля-фон Неймана, описывающего эволюцию функции распределения (статистического оператора) $F(t)$ всей рассматриваемой системы:

$$
\frac{\partial}{\partial t} F(t)=L(t) F(t)
$$

где $L(t)$ - эволюционный (супер)оператор Лиувилля, действующий на $F(t)$ и, вообще говоря, зависящий от времени. Функция распределения (или статистический оператор) подчинена условиям нормировки

$$
\int d x_{1} \ldots \int d x_{N} F\left(x_{1}, \ldots, x_{N}, t\right)=1
$$

или

$$
\operatorname{Tr} F(t)=1 \text {. }
$$

Формальное решение уравнения (6) можно записать как

$$
F(t)=U\left(t, t_{0}\right) F\left(t_{0}\right), \quad U\left(t, t_{0}\right)=T \exp \left[\int_{t_{0}}^{t} d s L(s)\right],
$$

где $T$ - оператор хронологического упорядочения по времени, который упорядочивает произведение зависящих от времени операторов по возрастанию их временны́х аргументов справа налево, а $F\left(t_{0}\right)$ - значение функции распределения в некоторый начальный момент времени $t_{0}$.

Введем зависящий от времени оператор $P(t)$, преобразующий функцию распределения $F(t)$ в релевантную функцию распределения $f_{\mathrm{r}}(t)$, которая, как правило, является вакуумной (без корреляций) многочастичной функцией распределения, т.е. произведением редуцированных функций распределения, необходимых и достаточных для описания эволюции измеряемых (статистических средних) значений.

Задача заключается в том, чтобы получить замкнутое эволюционное уравнение для редуцированных функций распределения, учитывая существование корреляций между элементами рассматриваемой системы, в том числе и корреляций в начальный момент времени $t_{0}$.

Таким образом, мы определяем релевантную функцию распределения как

$$
f_{\mathrm{r}}(t)=P(t) F(t)=P(t) U\left(t, t_{0}\right) F\left(t_{0}\right) .
$$

Заметим, что, вообще говоря, оператор $P(t)$ не является проекционным. Более того, действие оператора $P(t)$ на $F(t)$ более не является линейной операцией (в отличие 
от применения обычного не зависящего от времени проекционного оператора [9]), поскольку $P(t)$ может зависеть от зависящей от времени рассматриваемой функции распределения (см. ниже). Тем самым, применяя $P(t)$ к линейному уравнению Лиувилля (6), получаем в общем случае нелинейное уравнение. Оператор $P(t)$ не коммутирует с производной $\partial / \partial t$ (не зависящие от времени линейные проекционные операторы коммутируют с $\partial / \partial t)$ и $P\left(t^{\prime}\right) F(t) \neq f_{\mathrm{r}}(t)\left(\right.$ при $\left.t^{\prime} \neq t\right)$.

Используя уравнение Лиувилля-фон Неймана (6), уравнение движения для $f_{\mathrm{r}}(t)$ (10) можно записать в виде

$$
\frac{\partial f_{\mathrm{r}}(t)}{\partial t}=\left[\frac{\partial P(t)}{\partial t}+P(t) L(t)\right]\left[f_{\mathrm{r}}(t)+f_{\mathrm{i}}(t)\right]
$$

где $f_{\mathrm{i}}(t)=\left[F(t)-f_{\mathrm{r}}(t)\right]$, а $\partial P(t) / \partial t-$ оператор, полученный взятием производной оператора $P(t)$, т.е. предполагается, что оператор $\partial P(t) / \partial t$ существует. Чтобы придать смысл производной $\partial P(t) / \partial t$, можно определить $P(t)$ как

$$
P(t)=C(t) D
$$

где $C(t)$ - хорошо определенная (операторная) функция времени, а $D$ - не зависящий от времени (супер)оператор, действующий на $F(t)$ и реализующий интегрирование по всем лишним переменным в духе метода сокращенного описания (конкретизация оператора $P(t)$ будет более детально рассмотрена ниже).

Как следует из выражения (11), уравнение для нерелевантной части функции распределения $f_{\mathrm{i}}(t)$ можно получить, используя уравнения (6) и (11) и разбивая $F(t)$ на релевантную и нерелевантную части. Таким образом,

$$
\frac{\partial f_{\mathrm{i}}(t)}{\partial t}=\left[Q(t) L(t)-\frac{\partial P(t)}{\partial t}\right]\left[f_{\mathrm{i}}(t)+f_{\mathrm{r}}(t)\right]
$$

где $Q(t)=1-P(t)$, и поэтому $f_{\mathrm{i}}(t)=Q(t) F(t)$.

Формальное решение уравнения (13) имеет вид

$$
f_{\mathrm{i}}(t)=\int_{t_{0}}^{t} d t^{\prime} S\left(t, t^{\prime}\right)\left[Q\left(t^{\prime}\right) L\left(t^{\prime}\right)-\frac{\partial P\left(t^{\prime}\right)}{\partial t^{\prime}}\right] f_{\mathrm{r}}\left(t^{\prime}\right)+S\left(t, t_{0}\right) f_{\mathrm{i}}\left(t_{0}\right),
$$

где

$$
S\left(t, t_{0}\right)=T \exp \left\{\int_{t_{0}}^{t} d s\left[Q(s) L(s)-\frac{\partial P(s)}{\partial s}\right]\right\} .
$$

Последний оператор можно представить в виде ряда:

$$
\begin{aligned}
S\left(t, t_{0}\right)=1 & +\int_{t_{0}}^{t} d t_{1}\left[Q\left(t_{1}\right) L\left(t_{1}\right)-\frac{\partial P\left(t_{1}\right)}{\partial t_{1}}\right]+ \\
& +\int_{t_{0}}^{t} d t_{1} \int_{t_{1}}^{t} d t_{2}\left[Q\left(t_{2}\right) L\left(t_{2}\right)-\frac{\partial P\left(t_{2}\right)}{\partial t_{2}}\right]\left[Q\left(t_{1}\right) L\left(t_{1}\right)-\frac{\partial P\left(t_{1}\right)}{\partial t_{1}}\right]+\cdots
\end{aligned}
$$

Заметим, что если операторы $P$ и $L$ не зависят от времени, то $S\left(t, t_{0}\right)=e^{Q L\left(t-t_{0}\right)}$. 
Подставляя (14) в (11), получаем

$$
\begin{aligned}
\frac{\partial f_{\mathrm{r}}(t)}{\partial t}= & {\left[P(t) L(t)+\frac{\partial P(t)}{\partial t}\right]\left\{f_{\mathrm{r}}(t)+\int_{t_{0}}^{t} d t^{\prime} S\left(t, t^{\prime}\right)\left[Q\left(t^{\prime}\right) L\left(t^{\prime}\right)-\frac{\partial P\left(t^{\prime}\right)}{\partial t^{\prime}}\right] f_{\mathrm{r}}\left(t^{\prime}\right)+\right.} \\
& \left.+S\left(t, t_{0}\right) f_{\mathrm{i}}\left(t_{0}\right)\right\} .
\end{aligned}
$$

Уравнение (17) представляет собой обобщение ОУУ Накаджимы-Цванцига со сверткой по времени (см. [8]-[10]) для релевантной части функции распределения на случай зависящих от времени операторов $P$ и $Q$. Как уже было упомянуто, операторы $P(t)$ и $Q(t)$ не являются, вообще говоря, проекционными в обычном смысле, и в нашем выводе уравнения (17) не использовалось такое свойство проекционных операторов, как $P^{2}=P, Q^{2}=Q$ (как будет показано ниже, для $P(t) P\left(t^{\prime}\right)$ и $Q(t) Q\left(t^{\prime}\right)$ такое свойство имеет место только при $\left.t=t^{\prime}\right)$. Полученное уравнение, как и обычное ОУУ со сверткой по времени, является точным неоднородным интегро-дифференциальным уравнением для релевантной части функции распределения (статистического оператора), содержащим нерелевантную часть функции распределения (источник) в начальный момент времени $f_{\mathrm{i}}\left(t_{0}\right)=F\left(t_{0}\right)-f_{\mathrm{r}}\left(t_{0}\right)$. Однако уравнение (17), вообще говоря, эквивалентно нелинейному уравнению для интересующей нас редуцированной функции распределения (например, для одночастичной функции распределения, которую мы рассмотрим в следующем разделе), и поэтому оно также удобно для изучения эволюции многочастичных систем, которые описываются нелинейными уравнениями типа уравнения Больцмана (в кинетическом режиме) и в которых нелинейность обусловлена столкновениями между частицами.

Для не зависящих от времени операторов $P$ и $Q$ уравнение (17) редуцируется к обычному линейному ОУУ со сверткой по времени. Уравнения такого типа больше подходят для исследования, например, эволюции подсистемы, взаимодействующей с большой системой в состоянии теплового равновесия (термостатом).

Для упрощения уравнения (17) и придания смысла производной $\partial P(t) / \partial t$ рассмотрим оператор $P(t)$ вида (12). Такое представление оператора $P(t)$ подходит для исследования многих интересных случаев (см. раздел 3 ). Кроме того, разбиение функции распределения на релевантную часть $P(t) F(t)$ и нерелевантную часть $Q(t) F(t)$ имеет смысл, если оператор $P(t)$ удовлетворяет соотношению

$$
P(t) P(t)=P(t)
$$

для любого момента времени $t$. Тогда, если $P(t)=C(t) D$, то из уравнения (18) следует, что

$$
D C(t)=1
$$

для любого момента времени $t$. Уравнение (19), вообе говоря, представляет собой условие нормировки для функций распределения, составляющих $C(t)($ см. (7), (8) и (36) ниже), и из него следует, что $C(t)$ зависит от переменных, которые удалены из функции распределения $F(t)$ посредством оператора $D$ (как видно из (3), 
$f_{\mathrm{r}}(t)=P(t) F(t)$ зависит от полного набора переменных функции распределения $F(t))$. Из свойства (19) получаем

$$
\begin{gathered}
P(t) P\left(t^{\prime}\right)=P(t), \quad Q(t) Q\left(t^{\prime}\right)=Q\left(t^{\prime}\right), \quad P(t) Q\left(t^{\prime}\right)=0, \\
Q\left(t^{\prime}\right) P(t)=P(t)-P\left(t^{\prime}\right) .
\end{gathered}
$$

Условие (19) приводит также к следующим соотношениям для производной оператора $P(t)$ по времени:

$$
D \frac{\partial P(t)}{\partial t}=D \frac{\partial C(t)}{\partial t} D=0
$$

где мы учли, что операторы $D$ и $\partial / \partial t$ коммутируют ( $D$ не зависит от времени).

Введем также редуцированную функцию распределения

$$
f_{\text {red }}(t)=D F(t)=D f_{\mathrm{r}}(t)
$$

которая потребуется для вычисления интересующих нас ожидаемых значений в неравновесном состоянии. Ввиду (19) имеем

$$
D \frac{\partial f_{\mathrm{r}}(t)}{\partial t}=\frac{\partial f_{\mathrm{red}}(t)}{\partial t}, \quad D f_{\mathrm{i}}(t)=D Q(t) F(t)=0 .
$$

Таким образом, применяя оператор $D$ к уравнению (17) слева, получаем

$$
\frac{\partial f_{\text {red }}(t)}{\partial t}=D L(t)\left\{f_{\mathrm{r}}(t)+\int_{t_{0}}^{t} d t^{\prime} S\left(t, t^{\prime}\right)\left[Q\left(t^{\prime}\right) L\left(t^{\prime}\right)-\frac{\partial P\left(t^{\prime}\right)}{\partial t^{\prime}}\right] f_{\mathrm{r}}\left(t^{\prime}\right)+S\left(t, t_{0}\right) f_{\mathrm{i}}\left(t_{0}\right)\right\},
$$

где использовались соотношения (19), (21) и (23).

Без потери общности гамильтониан системы можно разбить на два слагаемых: $H(t)=H^{0}(t)+H^{\prime}(t)\left(L(t)=L^{0}(t)+L^{\prime}(t)\right)$, где $H^{0}\left(L^{0}\right)$ связан с энергией невзаимодействующих частиц (или подсистем), а $H^{\prime}\left(L^{\prime}\right)$ описывает взаимодействие между частицами (подсистемами). Тогда, кроме удовлетворения условию (19), оператор $D$, посредством которого выбирается редуцированная функция распределения, должен коммутировать с оператором $L^{0}$ следующим образом:

$$
D L^{0}(t)=L_{\text {red }}^{0}(t) D
$$

где $L_{\mathrm{red}}^{0}(t)$ - редуцированный оператор Лиувилля $L^{0}$, зависящий только от переменных редуцированной функции распределения $f_{\text {red }}(t)$. Выражение $(25)$ означает, что обе его части действуют на произвольную функцию (оператор), определенную на фазовом (гильбертовом) пространстве. Учитывая, что $C\left(t^{\prime}\right) L_{\mathrm{red}}^{0}(t)=L_{\mathrm{red}}^{0}(t) C\left(t^{\prime}\right)$, можно записать уравнение (25) в более общем виде:

$$
P\left(t^{\prime}\right) L^{0}(t)=L_{\text {red }}^{0}(t) P\left(t^{\prime}\right)
$$

$\left(C\left(t^{\prime}\right)\right.$ зависит от набора переменных, которые удалены из $F(t)$ с помощью оператора $D$, а $L_{\text {red }}^{0}(t)$ не зависит от них). Коммутационное соотношение (25) следует 
из соображений самосогласованности, поскольку если $L^{\prime}(t)=0$, то эволюция “свободной” (не взаимодействующей с остальной системой) подсистемы должна описываться уравнением (24) только посредством $L_{\text {red }}^{0}(t)\left(D L^{0}(t) f_{\mathrm{r}}(t)=L_{\mathrm{red}}^{0}(t) f_{\mathrm{red}}(t)=\right.$ $L_{\mathrm{red}}^{0}(t) D f_{\mathrm{r}}(t)$, см. также [1]). В этом можно убедиться исходя из соотношений

$$
\begin{aligned}
& D L^{0}(t) S\left(t, t_{1}\right) Q\left(t_{2}\right)=0, \\
& D L^{0} S\left(t, t_{1}\right) \frac{\partial P\left(t_{2}\right)}{\partial t_{2}}=0,
\end{aligned}
$$

которые следуют из $(25),(15),(21)$ и $(23)$. Таким образом, все члены в $(24)$, кроме первого $\left(L_{\text {red }}^{0}(t) f_{\text {red }}(t)\right)$, пропорциональны $L^{\prime}(t)$.

Аналогично, используя (25), (15) и соотношения, следующие из (19), можно доказать равенства

$$
\begin{gathered}
S\left(t, t_{1}\right) Q\left(t_{2}\right)=\bar{U}\left(t, t_{1}\right) Q\left(t_{2}\right) \\
S\left(t, t_{1}\right) \frac{\partial P\left(t_{2}\right)}{\partial t_{2}}=\bar{U}\left(t, t_{1}\right) \frac{\partial P\left(t_{2}\right)}{\partial t_{2}}
\end{gathered}
$$

где

$$
\bar{U}\left(t, t_{1}\right)=T \exp \left\{\int_{t_{1}}^{t} d s\left[L^{0}(s)+Q(s) L^{\prime}\right]\right\} .
$$

Таким образом, используя (27) и (28) в (24), можно окончательно переписать уравнение (17) как следующее уравнение для релевантной части функции распределения (статистического оператора):

$$
\begin{aligned}
\frac{\partial D f_{\mathrm{r}}(t)}{\partial t}= & D L(t) f_{\mathrm{r}}(t)+D L^{\prime}(t) \int_{t_{0}}^{t} d t^{\prime} \bar{U}\left(t, t^{\prime}\right)\left[Q\left(t^{\prime}\right) L\left(t^{\prime}\right)-\frac{\partial P\left(t^{\prime}\right)}{\partial t^{\prime}}\right] f_{\mathrm{r}}\left(t^{\prime}\right)+ \\
& +D L^{\prime}(t) \bar{U}\left(t, t_{0}\right) f_{\mathrm{i}}\left(t_{0}\right),
\end{aligned}
$$

где $D f_{\mathrm{r}}(t)=f_{\text {red }}(t)$, причем выполнено соотношение $(25)$.

В следующем разделе будет показано, что основные соотношения (19) и (25) выполняются для газа взаимодействующих классических частиц.

\section{3. РАЗРЕЖЕННЫЙ НЕОДНОРОДНЫЙ ГАЗ КЛАССИЧЕСКИХ ЧАСТИЦ}

Применим уравнение (30) к случаю газа из $N(N \gg 1)$ тождественных классических частиц. Оператор Лиувилля $L$ для такой системы можно представить в виде

$$
\begin{aligned}
L & =L^{0}+L^{\prime}, \\
L^{0} & =\sum_{i=1}^{N} L_{i}^{0}, \quad L_{i}^{0}=-\mathbf{v}_{i} \cdot \nabla_{i}, \quad \mathbf{v}_{i}=\frac{\mathbf{p}_{i}}{m}, \quad \nabla_{i}=\frac{\partial}{\partial \mathbf{x}_{i}}, \\
L^{\prime} & =\sum_{\substack{i, j=1 \\
i<j}}^{N} L_{i j}^{\prime}, \quad L_{i j}^{\prime}=\left(\nabla_{i} V_{i j}\right) \cdot\left(\frac{\partial}{\partial \mathbf{p}_{i}}-\frac{\partial}{\partial \mathbf{p}_{j}}\right),
\end{aligned}
$$


где $L^{0}$ соответствует кинетической энергии $H^{0}=\sum_{i=1}^{N} \mathbf{p}_{i}^{2} / 2 m$ частицы с импульсами $\mathbf{p}_{i}$ и массой $m$, а $L^{\prime}$ соответствует взаимодействию между частицами $H^{\prime}=$ $\sum_{i, j=1, i<j}^{N} V_{i j}$ с парным потенциалом $V_{i j}=V\left(\left|\mathbf{x}_{i}-\mathbf{x}_{j}\right|\right)$. Таким образом, в рассматриваемом случае оператор $L$ не зависит от времени. Здесь мы не предполагаем взаимодействие слабым, но считаем, что выполнены все необходимые требования к свойствам сил, посредством которых частицы взаимодействуют друг с другом (в частности, не образуется связанных состояний).

Кроме того, мы, как обычно, полагаем, что все определенные на фазовом пространстве функции $\Phi\left(x_{1}, \ldots, x_{N}\right)$ и их производные обращаются в нуль на границах конфигурационного пространства и при $\mathbf{p}_{i}= \pm \infty$. Эти граничные условия и явный вид операторов Лиувилля (13) приводят к соотношениям

$$
\begin{gathered}
\int d x_{i} L_{i}^{0} \Phi\left(x_{1}, \ldots, x_{N}, t\right)=0 \\
\int d x_{i} \int d x_{j} L_{i j}^{\prime} \Phi\left(x_{1}, \ldots, x_{N}, t\right)=0 .
\end{gathered}
$$

Мы ищем эволюционное уравнение для одночастичной функции распределения

$$
F_{1}\left(x_{i}, t\right)=V \int d x_{1} \ldots \int d x_{i-1} \int d x_{i+1} \ldots \int d x_{N} F\left(x_{1}, \ldots, x_{N}, t\right),
$$

где $V$ - объем системы. Для этого удобно определить оператор $P(t)$ в виде

$$
P(t)=C(t) D, \quad C(t)=\left[\prod_{i=2}^{N} F_{1}\left(x_{i}, t\right)\right], \quad D=\frac{1}{V^{N-1}} \int d x_{2} \ldots \int d x_{N} .
$$

Вообще говоря, $s$-частичная $(s \leqslant N)$ функция распределения определяется как

$$
F_{s}\left(x_{1}, \ldots, x_{s}, t\right)=V^{s} \int d x_{s+1} \ldots \int d x_{N} F\left(x_{1}, \ldots, x_{N}, t\right)
$$

и удовлетворяет условию нормировки (см. (7))

$$
\int d x_{1} \ldots \int d x_{s} F_{s}\left(x_{1}, \ldots, x_{s}, t\right)=V^{s} .
$$

Поскольку функция распределения (35) для $s=N, F_{N}(t)=V^{N} F(t)$ (здесь и ниже зависимость от $x_{i}$ часто опускается для краткости записи), удовлетворяет уравнению Лиувилля (6), можно применить проекционный оператор $(34)$ к $F_{N}(t)$ (вместо $F(t))$ и получить следующую релевантную часть функции распределения:

$$
f_{\mathrm{r}}(t)=P(t) F_{N}(t)=\prod_{i=1}^{N} F_{1}\left(x_{i}, t\right) .
$$

Из определения (37) видно преимущество зависящего от времени оператора $P(t)$ : применяя его к $N$-частичной функции распределения $F_{N}(t)$, можно определить релевантную часть (37) функции распределения, которая выглядит более естественно 
и симметрично, чем выражение (5), использованное в работе [15] и полученное с помощью не зависящего от времени проекционного оператора (4) (нам пришлось тогда воспользоваться не зависящим от времени проекционным оператором, поскольку рассмотренное в работе [15] ОУУ со сверткой по времени справедливо только для таких операторов). Более того, релевантная часть (37) имеет ясный физический смысл, поскольку она представляет собой медленно меняющуюся вакуумную (без корреляций) часть $N$-частичной функции распределения, которая главным образом и интересна.

Таким образом, нерелевантная часть

$$
f_{\mathrm{i}}(t)=Q(t) F_{N}(t)=F_{N}(t)-\prod_{i=1}^{N} F_{1}\left(x_{i}, t\right)
$$

описывает все межчастичные корреляции, в частности начальные корреляции, которые составляют неоднородный член в уравнении (30). Нерелевантную часть $N$-частичной функции распределения в момент времени $t_{0}$ (а также и в любой другой момент времени $t) f_{\mathrm{i}}\left(t_{0}\right)=F_{N}\left(t_{0}\right)-f_{\mathrm{r}}\left(t_{0}\right)$ всегда можно представить в виде кластерного разложения:

$$
f_{\mathrm{i}}\left(t_{0}\right)=\sum_{\substack{i, j=1, i<j}}^{N} g_{2}\left(x_{i}, x_{j}\right) \prod_{\substack{k=1, k \neq i, j}}^{N-2} F_{1}\left(x_{k}\right)+\sum_{\substack{i, j, k=1, i<j<k}}^{N} g_{3}\left(x_{i}, x_{j}, x_{k}\right) \prod_{\substack{l=1, l \neq i, j, k}}^{N-3} F_{1}\left(x_{l}\right)+\cdots,
$$

где $\prod_{k=1, k \neq i, j}^{N-2} F_{1}\left(x_{k}\right)$ и $\prod_{l=1, l \neq i, j, k}^{N-3} F_{1}\left(x_{l}\right)$ обозначают произведения $N-2$ и $N-3$ одночастичных функций распределения с $k \neq i, j$ и $l \neq i, j, k$ соответственно, а $g_{2}\left(x_{i}, x_{j}\right)$ и $g_{3}\left(x_{i}, x_{j}, x_{k}\right)$ - неприводимые двухчастичные и трехчастичные корреляционные функции (остальные члены в выражении (39) определяются аналогично). Здесь и далее одночастичные функции распределения и корреляционные функции с опущенным временны́м аргументом обозначают функции, взятые в момент времени $t=t_{0}$ (например, $\left.F_{1}\left(x_{k}\right)=F_{1}\left(x_{k}, t_{0}\right)\right)$.

Используя условие нормировки (36) для одночастичной функции распределения $\int F_{1}\left(x_{i}, t\right) d x_{i}=V$, легко видеть, что применяя интегральный оператор $D$ к $C(t)$ (оба эти объекта определяются в $(34)$ ), мы получаем $D C(t)=1$, т.е. выполняется условие (19) и его следствия, определяемые выражениями (20) и (21). Кроме того, в этом случае

$$
\begin{gathered}
f_{\text {red }}(t)=D f_{\mathrm{r}}(t)=F_{1}\left(x_{1}, t\right), \\
D Q(t)=0, \quad D f_{\mathrm{i}}(t)=0
\end{gathered}
$$

в соответствии с $(22)$ и $(23)$.

Правила коммутации (25) и (26) также следуют из (31), (32) и (34), поскольку

$$
D L^{0}=L_{1}^{0} D, \quad P(t) L^{0}=L_{1}^{0} P(t),
$$

т.е. в рассматриваемом случае $L_{\text {red }}^{0}=L_{1}^{0}$. Поэтому выполняются также равенства (27)-(29), где $L^{0}$ и $L^{\prime}$ в рассматриваемом случае не зависят от времени. 
Используя (32), (40) и (41), уравнение (30) можно представить в рассматриваемом случае как

$$
\begin{aligned}
\frac{\partial F_{1}\left(x_{1}, t\right)}{\partial t}= & L_{1}^{0} F_{1}\left(x_{1}, t\right)+n \int d x_{2} L_{12}^{\prime} F_{1}\left(x_{2}, t\right) F_{1}\left(x_{1}, t\right)+ \\
& +D L^{\prime} \int_{t_{0}}^{t} d t^{\prime} \bar{U}\left(t, t^{\prime}\right)\left[\left(L^{0}-L_{1}^{0}\right)+Q\left(t^{\prime}\right) L^{\prime}-\frac{\partial P\left(t^{\prime}\right)}{\partial t^{\prime}}\right] f_{\mathrm{r}}\left(t^{\prime}\right)+ \\
& +D L^{\prime} \bar{U}\left(t, t_{0}\right) f_{\mathrm{i}}\left(t_{0}\right)
\end{aligned}
$$

где $n=N / V$ - плотность частиц, а $D, f_{\mathrm{r}}(t)$ и $f_{\mathrm{i}}\left(t_{0}\right)$ соответственно определяются выражениями (34), (37) и (39). Эволюционный оператор (29) можно представить в виде ряда, аналогичного (16):

$$
\begin{aligned}
\bar{U}\left(t, t^{\prime}\right)=1 & +\int_{t^{\prime}}^{t} d t_{1}\left[L^{0}+Q\left(t_{1}\right) L^{\prime}\right]+ \\
& +\int_{t^{\prime}}^{t} d t_{1} \int_{t_{1}}^{t} d t_{2}\left[L^{0}+Q\left(t_{2}\right) L^{\prime}\right]\left[L^{0}+Q\left(t_{1}\right) L^{\prime}\right]+\cdots
\end{aligned}
$$

Рассмотрим уравнение (42) в первом приближении по плотности частиц $n$. Соответствующий безразмерный малый параметр разложения в теории возмущений

$$
\gamma=r_{0}^{3} n \ll 1
$$

где $r_{0}$ - эффективный радиус межчастичного взаимодействия. Из второго уравнения (32) легко видеть, что в разложении (43) все члены, содержащие $P(t) L^{\prime}$, пропорциональны по крайней мере первой степени плотности $n$ (как и второй член в правой части выражения (42)). Таким образом, в первом приближении по $n$ можно пренебречь всеми членами разложения (43), содержащими $P(t) L^{\prime}$, поскольку члены уравнения (42), содержащие $\bar{U}\left(t, t^{\prime}\right)$, уже имеют первый порядок по $n$ (применение $D L^{\prime}$ приводит к выражению по крайней мере первого порядка по $\left.n\right)$. В данном приближении все члены под интегралами в выражении (43) не зависят от времени, и оператор $\bar{U}\left(t, t^{\prime}\right)$ сводится к оператору вида

$$
\bar{U}\left(t, t^{\prime}\right)=e^{\left(L^{0}+L^{\prime}\right)\left(t-t^{\prime}\right)} .
$$

Теперь удобно использовать следующее разложение экспоненциального оператоpa (45):

$$
e^{\left(L^{0}+L^{\prime}\right)\left(t-t^{\prime}\right)}=e^{L^{0}\left(t-t^{\prime}\right)}+\int_{t^{\prime}}^{t} d \theta e^{L^{0}(t-\theta)} L^{\prime} e^{\left(L^{0}+L^{\prime}\right) \theta}
$$


Используя это разложение и соотношения (32), получаем из (42) следующее эволюционное уравнение для одночастичной функции распределения в линейном приближении по параметру плотности (44):

$$
\begin{aligned}
& \frac{\partial F_{1}\left(x_{1}, t\right)}{\partial t}=L_{1}^{0} F_{1}\left(x_{1}, t\right)+n \int d x_{2} L_{12}^{\prime} F_{1}\left(x_{2}, t\right) F_{1}\left(x_{1}, t\right)+ \\
& \quad+n \int d x_{2} L_{12}^{\prime} \int_{0}^{t-t_{0}} d t_{1} e^{\left(L_{12}^{0}+L_{12}^{\prime}\right) t_{1}}\left[\left(L_{2}^{0}+L_{12}^{\prime}\right) F_{1}\left(x_{2}, t-t_{1}\right) F_{1}\left(x_{1}, t-t_{1}\right)+\right. \\
& \left.\quad+\frac{\partial F_{1}\left(x_{2}, t-t_{1}\right)}{\partial t_{1}} F_{1}\left(x_{1}, t-t_{1}\right)\right]+n \int d x_{2} L_{12}^{\prime} e^{\left(L_{12}^{0}+L_{12}^{\prime}\right)\left(t-t_{0}\right)} g_{2}\left(x_{1}, x_{2}\right)
\end{aligned}
$$

где $L_{12}^{0}=L_{1}^{0}+L_{2}^{0}$. При получении (47) также использовалось определение нерелевантной части функции распределения (39) и учитывалось, что каждое дополнительное интегрирование по $x_{3}, \ldots$ добавляет лишнюю степень по $n$ (поэтому в линейном приближении по $n$ во все формулы входит не более одного интегрирования по фазовому пространству).

Уравнение (47) представляет собой основной результат данного раздела. Следует подчеркнуть, что соотношение (47) является нелинейным неоднородным немарковским управляющим уравнением со сверткой по времени для одночастичной функции распределения, содержащим начальные корреляции (источник). Заметим, что мы строго вывели это новое уравнение (обычно требуемое нелинейное управляющее уравнение постулируется [11]). Уравнение (47) является точным в линейном приближении по малому параметру плотности (44) и справедливо на любом временно́м масштабе и для любой рассматриваемой пространственно неоднородной системы. Первый член в правой части представляет собой обычный член с потоком. Второе слагаемое - это нелинейный власовский член, представляющий самосогласованное поле, действующее на данную (первую) частицу и определяемое всеми частицами системы. Важно подчеркнуть, что этот член (проявляющийся только в пространственно неоднородном случае) невозможно (без дополнительных приближений) получить из ОУУ (или нелинейного ОУУ), содержащего не зависящий от времени проекционный оператор (4) (см. [15]). Квадратичный по $L_{12}^{\prime}$ член описывает столкновения между частицами и приводит к диссипации в системе. Члены с $L_{2}^{0}$ (с пространственной производной) и с временно́й производной учитывают пространственные и временнь́е изменения одночастичной функции распределения на микроскопических (см. также ниже) масштабах порядка $r_{0}$ и $t_{\text {сог }} \sim r_{0} / \bar{v}$ соответственно. Эти два члена определяют вклад парных столкновений в недиссипативные характеристики (термодинамические функции) неидеального газа (см., например, [4]) и обычно отсутствуют при стандартном (на основе цепочки ББГКИ) выводе кинетических уравнений в линейном по $n$ приближении. Учет этих членов связан с некоторой проблемой, поскольку они обычно появляются в следующем (втором) приближении по $n$ (см., например, [17]), которое не согласуется с появляющимся в этом приближении диссипативным членом, определяемым трехчастичными столкновениями. Последний (нерелевантный) член в правой части (47) учитывает начальные корреляции (при 
$\left.t=t_{0}\right)$ и в линейном по $n$ приближении имеет вид двухчастичной корреляционной функции $g_{2}\left(x_{1}, x_{2}\right)$.

Рассмотрим члены в выражении (47) более внимательно, особенно в отношении их поведения с течением времени. Временна́я эволюция определяется точным двухчастичным пропагатором $G_{12}(t)=e^{\left(L_{12}^{0}+L_{12}^{\prime}\right) t}$ (это естественно для рассматриваемого разреженного газа в низшем порядке по плотности), который удовлетворяет интегральному уравнению

$$
G_{12}(t)=G_{12}^{0}(t)+\int_{0}^{t} d t_{1} G_{12}^{0}\left(t-t_{1}\right) L_{12}^{\prime} G_{12}\left(t_{1}\right),
$$

где $G_{12}^{0}(t)=e^{L_{12}^{0} t}-$ пропагатор невзаимодействующих частиц. Как следует из (31), действие пропагатора $G_{12}^{0}(t)$ на любую функцию, определенную на фазовом пространстве, имеет вид

$$
\begin{aligned}
e^{L^{0} t} \Phi\left(x_{1}, \ldots, x_{N}, t\right) & =\left[\prod_{i=1}^{N} e^{L_{i}^{0} t}\right] \Phi\left(x_{1}, \ldots, x_{N}, t\right)= \\
& =\Phi\left(\mathbf{x}_{1}-\mathbf{v}_{1} t, \mathbf{p}_{1}, \ldots, \mathbf{x}_{N}-\mathbf{v}_{N} t, \mathbf{p}_{N}, t\right) .
\end{aligned}
$$

Если динамика частиц обладает необходимыми свойствами, такими как, например, перемешивающий эргодический поток в фазовом пространстве, возникающий вследствие локальной (стохастической) неустойчивости, то под действием пропагатора $G_{12}(t)$ расстояния между частицами будут быстро (экспоненциально) расти со временем (вклад "параллельного движения" пренебрежимо мал). Было доказано, что такое поведение имеет место, например, для больцмановского разреженного газа, состоящего из твердых сфер, и в настоящее время считается характерным для большинства реальных систем, рассматриваемых в статистической физике (см., например, [4]). Перемешивающий эргодический поток в фазовом пространстве приводит к экспоненциальному затуханию корреляций и является причиной необратимости. Таким образом, если эффективное взаимодействие между частицами $V\left(\left|\mathbf{r}_{i}-\mathbf{r}_{j}\right|\right)$ обращается в нуль на расстоянии $\left|\mathbf{r}_{i}-\mathbf{r}_{j}\right|>r_{0}$, то выражение, стоящее под знаком интеграла по $t_{1}$ в уравнении (47), обращается в нуль при $t-t_{0}>t_{\text {cor }}$, и то же верно (при действии пропагатора $\left.G_{12}\left(t-t_{0}\right)\right)$ для члена, соответствующего начальным корреляциям, определяемым функцией $g_{2}\left(x_{1}, x_{2}\right)$, которая также зависит от взаимодействия между частицами (более подробно это обсуждается ниже, а пространственно однородный случай был рассмотрен в работе [15]).

Из условия (44) следует существование временно́й иерархии:

$$
t_{\text {cor }} \ll t_{\text {rel }},
$$

где $t_{\text {rel }} \sim \gamma^{-1} t_{\text {cor }}$ - время релаксации для одночастичной функции распределения $F_{1}\left(x_{i}, t\right)\left(1 / n r_{0}^{2}\right.$ - средняя длина свободного пробега частицы). На начальном этапе эволюции $t_{0} \leqslant t \leqslant t_{\text {cor }}$, который очень интересен и важен для понимания проблемы необратимости, а также для изучения немарковских процессов (эффектов памяти), явлений декогеренции и сверхбыстрой релаксации, могут быть существенными начальные корреляции. Однако если рассматривать временно́й масштаб 
$t-t_{0} \gg t_{\text {cor }}$, то начальные корреляции затухают (в результате перемешивающего потока) и в уравнении (47) можно опустить источник, содержащий величину $g_{2}\left(x_{1}, x_{2}\right)$. Однако, как отметил Боголюбов в работе [1], это можно сделать только на временно́м интервале (1), который существует вследствие выполнения (50). Последнее заключение следует из того факта, что поправки к решению уравнения (47), обусловленные начальными корреляциями, становятся пропорциональными величине $\left|t-t_{0}\right|$ (секулярные члены) и являются малыми только при $\left|t-t_{0}\right| \ll t_{\text {rel }}$. Таким образом, разложение по $n$ нерелевантного члена в начальных условиях (аналогично тому, как мы это проделали при переходе от (42) к (47)) неэффективно на рассматриваемом временно́м масштабе $t-t_{0} \gtrsim t_{\text {rel }}$ в кинетической теории.

Чтобы избавиться от нежелательных членов в начальных условиях, часто предполагают $f_{\mathrm{i}}\left(t_{0}\right)=0$, что соответствует приближению случайных фаз и в принципе является некорректным (см. [7]). Поэтому, как уже упоминалось в разделе 1, для изучения кинетической стадии эволюции при $t \gtrsim t_{\text {rel }}$ требуется более изощренный подход, подобный тому, который основан на принципе ослабления начальных корреляций (анзац Боголюбова) [1]. Однако подход Боголюбова не позволяет рассматривать начальную стадию эволюции $t_{0} \leqslant t \leqslant t_{\text {сог }}$ и ситуации, в которых существенную роль могут играть крупномасштабные корреляции при $t_{\text {cor }} \gtrsim t_{\text {rel }}$.

Если $F_{1}\left(x_{i}, t\right)$ значительно изменяется на макроскопическом (гидродинамическом) масштабе $l_{h}$ с соответствующим временнь́м масштабом $t_{h}=l_{h} / \bar{v}$ (для пространственно однородного газа имеются только характерные масштабы $l$ и $t_{\mathrm{rel}}$, на которых изменяется $\left.F_{1}\left(x_{i}, t\right)\right)$, то в уравнении (47) можно пренебречь членами с $L_{2}^{0}$ и $\partial F_{1}\left(x_{2}, t-t^{\prime}\right) / \partial t^{\prime}$, поскольку оба они имеют по крайней мере порядок $\gamma$, т.е. их вклад - величина второго порядка по $n$ (однако эти члены нельзя опустить, если мы хотим самосогласованно учитывать недиссипативные характеристики неидеального газа). На кинетическом временно́м масштабе $t-t_{0} \gtrsim t_{\text {rel }}$ верхний предел интегрирования по $t_{1}$ можно продолжить до бесконечности $\left(t_{0} \rightarrow-\infty\right)$ из-за затухания корреляций, обусловленных столкновениями. Отнесение верхнего предела интегрирования на бесконечность и факт существования этого предела могут подразумевать некоторую процедуру огрубления (сглаживания). Если мы также примем приближение случайных фаз (или принцип ослабления начальных корреляций) при $t_{0} \rightarrow-\infty$, т.е. не будем учитывать член с $g_{2}\left(x_{1}, x_{2}\right)$ в начальных условиях, то уравнение (47) сведется к уравнению вида

$$
\begin{aligned}
\frac{\partial F_{1}\left(x_{1}, t\right)}{\partial t}= & L_{1}^{0} F_{1}\left(x_{1}, t\right)+n \int d x_{2} L_{12}^{\prime} F_{1}\left(x_{2}, t\right) F_{1}\left(x_{1}, t\right)+ \\
& +n \int d x_{2} L_{12}^{\prime} \int_{0}^{\infty} d t_{1} e^{\left(L_{12}^{0}+L_{12}^{\prime}\right) t_{1}} L_{12}^{\prime} F_{1}\left(x_{2}, t\right) F_{1}\left(x_{1}, t\right),
\end{aligned}
$$

где мы учли, что в рамках принятого приближения при $t \gtrsim t_{\text {rel }} \gg t_{\text {cor }}$ можно заменить $F_{1}\left(x_{i}, t-t_{1}\right)$ на $F_{1}\left(x_{i}, t\right)$ (интегрирование по $t_{1}$ в $(47)$ дает существенный вклад только до $\left.t_{1} \sim t_{\text {cor }}\right)$. В последнем (столкновительном) члене в урав- 
нении (51) функции распределения сталкивающихся частиц можно с принятой точностью $\sim r_{0} / r_{h} \ll 1$ (или $r_{0} / l \ll 1$ ) взять в той же точке пространства $\mathbf{r}_{1}=\mathbf{r}_{2}$.

Если последний интеграл в (51) существует (скажем, в смысле Абеля), то уравнение (51) представляет собой нелинейное необратимое во времени марковское кинетическое уравнение. В случае слабого взаимодействия между частицами это уравнение в первом приближении по малому параметру взаимодействия $\varepsilon$ дает обратимое уравнение Власова (уравнение (51) с отброшенным третьим столкновительным членом в правой части, который является членом второго порядка по $\varepsilon$ ). Во втором порядке по $\varepsilon$ это уравнение совпадает с кинетическим уравнением Власова-Ландау (см., например, [18]). Для пространственно однородного случая, когда одночастичная функция распределения не зависит от координат частиц, $F_{1}\left(x_{j}, t\right)=F_{1}\left(\mathbf{p}_{j}, t\right)$ $\left(\int F_{1}(\mathbf{p}, t) d \mathbf{p}=1\right)$, второй (власовский) член в уравнении (51) обращается в нуль, поскольку мы рассматриваем потенциал $V_{i j}(31)$, который зависит от разностей координат частиц. В этом случае уравнение (51) эквивалентно нелинейному уравнению Больцмана (см., например, [18]). Следует подчеркнуть, что в данном подходе (основанном на зависящем от времени операторе $P(t)(34)$ ), в отличие от подхода, основанного на не зависящем от времени проекционном операторе (см. [15], [16]), нелинейность уравнения (51) возникает совершенно естественным образом. В последнем из двух упомянутых подходов в эволюционном уравнении приходится заменять функцию $F_{1}\left(x_{2}, t_{0}\right)$ на ее приближение $F_{1}\left(x_{2}, t\right)$ (это возможно на временнь́х масштабах, задаваемых выражением (1)), чтобы получить нелинейное уравнение из линейного неоднородного ОУУ или однородного ОУУ. В случае подхода, основанного на использовании цепочки ББГКИ, нелинейность вводится через принцип ослабления начальных корреляций (2).

\section{4. ОДНОРОДНОЕ НЕЛИНЕЙНОЕ ОУУ С СОХРАНЕНИЕМ НАЧАЛЬНЫХ КОРРЕЛЯЦИЙ}

В разделе 2 было получено нелинейное ОУУ для релевантной части функции распределения (статистического оператора). Однако уравнение (17) не является замкнутым в том смысле, что оно является неоднородным и содержит нерелевантный член, включающий, вообще говоря, все многочастичные корреляции. Как было отмечено выше, чтобы избавиться от нерелевантной части и получить однородное уравнение (аналогичное уравнению (51)), например для одночастичной функции распределения, обычно используют принцип Боголюбова ослабления начальных корреляций или приближение случайных фаз.

Чтобы включить в рассмотрение начальные корреляции и получить эволюционные уравнения, справедливые на всех временнь́х масштабах, в работах [15], [16] был предложен метод, преобразующий обычные линейные неоднородные ОУУ в однородные ОУУ. Применим теперь этот подход к уравнению (30). Наша цель - получить из уравнения (30) однородное уравнение, содержащее нерелевантную часть функции распределения $f_{\mathrm{i}}\left(t_{0}\right)$ в "массовом" (супер)операторе, действующем на релевантную часть функции распределения $f_{\mathrm{r}}(t)$. Такое уравнение позволит учитывать 
начальные корреляции, содержащиеся в $f_{\mathrm{i}}\left(t_{0}\right)$, единообразно со всеми другими корреляциями (столкновениями) и будет справедливо на любом временно́м масштабе, включая начальную стадию эволюции, когда начальные корреляции заведомо важны. Это действительно так, поскольку не будет больше необходимости в ограничениях, задаваемых неравенствами (1), которые позволяют пренебречь начальными корреляциями и избежать появления "секулярных членов" в решении эволюционного уравнения (разложение по теории возмущений "массового" (супер)оператора, не приводит к возникновению таких членов на большом (кинетическом) временно́м масштабе). В отличие от однородных линейных ОУУ, полученных в работах [15], [16], выведенное ниже уравнение является нелинейным и также применимо к неконсервативным системам (с зависящими от времени гамильтонианами).

Следуя работе [15], тождественно представим нерелевантную часть функции распределения (статистического оператора) в виде

$$
\begin{aligned}
f_{\mathrm{i}}\left(t_{0}\right) & =F\left(t_{0}\right)-f_{\mathrm{r}}\left(t_{0}\right)=Q\left(t_{0}\right) F\left(t_{0}\right)= \\
& =\left[Q\left(t_{0}\right) F\left(t_{0}\right)\right] F^{-1}\left(t_{0}\right) U^{-1}\left(t, t_{0}\right)[P(t)+Q(t)] U\left(t, t_{0}\right) F\left(t_{0}\right)= \\
& =C_{0} U^{-1}\left(t, t_{0}\right)\left[f_{\mathrm{r}}(t)+f_{\mathrm{i}}(t)\right], \\
C_{0} & =\left[Q\left(t_{0}\right) F\left(t_{0}\right)\right] F^{-1}\left(t_{0}\right), \quad U^{-1}\left(t, t_{0}\right)=T_{-} \exp \left[-\int_{t_{0}}^{t} d s L(s)\right],
\end{aligned}
$$

где $U^{-1}\left(t, t_{0}\right)$ - оператор эволюции вспять по времени для матрицы плотности $F(t)$ $($ ср. с $(9)), U^{-1}\left(t, t_{0}\right) U\left(t, t_{0}\right)=1, T_{-}$- оператор антихронологического упорядочения по времени, упорядочивающий зависящие от времени операторы $L(s)$ по возрастанию временнь́х аргументов слева направо, $F^{-1}\left(t_{0}\right)$ - матрица, обратная $F\left(t_{0}\right)$, $F^{-1}\left(t_{0}\right) F\left(t_{0}\right)=1$, и $P(t)+Q(t)=1$. Таким образом, дополнительное тождество (52) получается путем умножения нерелевантной части на величину $F^{-1}\left(t_{0}\right) F\left(t_{0}\right)$, равную единице (при этом подразумевается существование $F^{-1}\left(t_{0}\right)$ ), и вставки величин $U^{-1}\left(t, t_{0}\right) U\left(t, t_{0}\right)=1$ и $P(t)+Q(t)=1$. Поэтому не может возникнуть ни расходимости (обусловленной возможным обращением в нуль матрицы $F\left(t_{0}\right)$ ), ни неопределенности типа 0/0 (числитель и знаменатель в отношении $F\left(t_{0}\right) / F\left(t_{0}\right)=1$ ведут себя аналогичным образом). Это справедливо для всех дальнейших (тождественных) преобразований (см. ниже).

В (52) введен следующий параметр начальных корреляций:

$$
\begin{aligned}
C_{0} & =\left[Q\left(t_{0}\right) F\left(t_{0}\right)\right] F^{-1}\left(t_{0}\right)=f_{\mathrm{i}}\left(t_{0}\right)\left[f_{\mathrm{r}}\left(t_{0}\right)+f_{\mathrm{i}}\left(t_{0}\right)\right]^{-1}= \\
& =f_{\mathrm{i}}\left(t_{0}\right) f_{\mathrm{r}}^{-1}\left(t_{0}\right)\left[1+f_{\mathrm{i}}\left(t_{0}\right) f_{\mathrm{r}}^{-1}\left(t_{0}\right)\right]^{-1}=\left(1-C_{0}\right) f_{\mathrm{i}}\left(t_{0}\right) f_{\mathrm{r}}^{-1}\left(t_{0}\right) .
\end{aligned}
$$

Важно отметить, что в выражениях (52) и (53) оператор $Q\left(t_{0}\right)$ действует только на $F\left(t_{0}\right)$, что отражено заключением величины $Q\left(t_{0}\right) F\left(t_{0}\right)$ в скобки. Это следует из того факта, что $f_{\mathrm{r}}(t)$ и $f_{\mathrm{i}}\left(t_{0}\right)$ являются основными величинами, с которыми 
мы имеем дело в уравнении (30). Все функции динамических переменных, средние значения которых можно вычислить с помощью $f_{\mathrm{r}}(t)\left(f_{\mathrm{red}}(t)\right)$, умножая уравнение (30) справа на соответствующие функции (операторы) и вычисляя среднее значение (след), зависят только от переменных, не удаленных содержащимся в $D$ интегрированием (оператор $D$ удаляет из $F(t)$ все избыточные переменные путем интегрирования). Поэтому, если мы представим $f_{\mathrm{r}}(t)$ и $f_{\mathrm{i}}\left(t_{0}\right)$ в $(30)$ как $f_{\mathrm{r}}(t)=P(t) F(t)$ и $f_{\mathrm{i}}\left(t_{0}\right)=Q\left(t_{0}\right) F\left(t_{0}\right)$, то проекционные операторы $P(t)=C(t) D$ и $Q(t)=1-P(t)$ в этих выражениях будут действовать только на $F(t)$, но не на функции (если таковые имеются), стоящие справа от них. В этом заключается суть метода редуцированного описания, когда для вычисления среднего значения функций, зависящих от гораздо меньшего числа переменных, чем полная функция распределения $F(t)$, нам в действительности требуется только редуцированная функция распределения (матрица плотности) $f_{\text {red }}(t)$.

Как видно из выражения (53), корреляционный параметр имеет вид ряда по $f_{\mathrm{i}}\left(t_{0}\right) f_{\mathrm{r}}^{-1}\left(t_{0}\right)$, поэтому нам может потребоваться только формальное существование функции $f_{\mathrm{r}}^{-1}\left(t_{0}\right)$, обратной к релевантной функции распределения, выбранной с помощью подходящего оператора $P\left(t_{0}\right)$. Представляется правдоподобным, что можно построить величину, обратную к релевантной части функции распределения, определенной в упомянутом выше смысле, т.е. к некоррелированной части (см. [15], [16] и следующий раздел).

Итак, имеются два уравнения, связывающие $f_{\mathrm{i}}(t)$ и $f_{\mathrm{i}}\left(t_{0}\right),-$ уравнения $(14)$ и $(52)$. Используя соотношения (28), выражая из этих уравнений $f_{\mathrm{i}}\left(t_{0}\right)$ как функцию $f_{\mathrm{r}}(t)$ и подставляя ее в выражение (30), получаем уравнение

$$
\begin{aligned}
\frac{\partial D f_{\mathrm{r}}(t)}{\partial t}= & D L^{0}(t) f_{\mathrm{r}}(t)+D L^{\prime}(t) R\left(t, t_{0}\right) f_{\mathrm{r}}(t)+ \\
& +D L^{\prime}(t) R\left(t, t_{0}\right) \int_{t_{0}}^{t} d t^{\prime} \bar{U}\left(t, t^{\prime}\right)\left[Q\left(t^{\prime}\right) L\left(t^{\prime}\right)-\frac{\partial P\left(t^{\prime}\right)}{\partial t^{\prime}}\right] f_{\mathrm{r}}\left(t^{\prime}\right),
\end{aligned}
$$

где оператор $R\left(t, t_{0}\right)$ определяется как

$$
\begin{aligned}
R\left(t, t_{0}\right) & =1+C\left(t, t_{0}\right), \\
C\left(t, t_{0}\right) & =\bar{U}\left(t, t_{0}\right)\left[1-C_{0}\left(t, t_{0}\right)\right]^{-1} C_{0} U^{-1}\left(t, t_{0}\right), \\
C_{0}\left(t, t_{0}\right) & =C_{0} U^{-1}\left(t, t_{0}\right) \bar{U}\left(t, t_{0}\right),
\end{aligned}
$$

$D f_{\mathrm{r}}(t)=f_{\text {red }}(t)$ и выполняется соотношение $(25)$.

Уравнение (54) является основным результатом настоящей работы. Мы вывели искомое однородное обобщенное эволюционное уравнение для релевантной части функции распределения (статистического оператора). Это уравнение отличается от линейного однородного ОУУ со сверткой по времени, полученного в работе [15] из обычного ОУУ Накаджимы-Цванцига с использованием техники не зависящего от времени проекционного оператора. Уравнение (54) нелинейно и справедливо для систем с зависящим от времени гамильтонианом. Оно справедливо как в классиче- 
ской, так и в квантовой физике, если произведено соответствующее переопределение символов и все (супер)операторы существуют (мы вернемся к этому вопросу ниже). При выводе уравнения (54) мы не делали никаких приближений, поэтому оно является точным интегро-дифференциальным уравнением, которое учитывает начальные корреляции и их динамику с помощью модифицированного (супер)оператора (функции памяти) из уравнения (30), действующего на релевантную часть функции распределения (статистического оператора) $f_{\mathrm{r}}(t)$.

Полученное точное ядро в уравнении (54) может служить отправной точкой для эффективных разложений по теории возмущений. Во многих случаях такие разложения однородных уравнений (типа уравнения (54)) справедливы в гораздо более широких пределах, нежели неоднородных (типа уравнения (30)), когда мы имеем дело скорее с разложениями функций $\left(f_{\mathrm{r}}, f_{\mathrm{i}}\right)$, а не уравнений (см. также [1]). В частности, можно ожидать, что расходимости, возникающие в членах высшего порядка в разложении по плотности газа [2], будут более удобным образом описываться "массовым" (супер)операторным разложением. Как видно из (54), временна́я эволюция начальных корреляций $\left(C\left(t, t_{0}\right)\right)$ влияет на интеграл столкновений, а также на второй член, что приводит к дополнительной зависимости от плотности $n$ (если используется разложение по $n$ ). Известно, что начальные корреляции в высших порядках по $n$ становятся более долгоживущими и затухают только при $t-t_{0} \sim t_{\mathrm{rel}}$ из-за последовательности парных столкновений (см. [2], [3]). В то время как начальные корреляции в низших порядках по $n$ (в приближении парных столкновений) являются типичными короткомасштабными корреляциями с характерными параметрами $t_{\text {cor }} \sim r_{0} / \bar{v} \ll t_{\text {rel }}$ и $l_{\text {cor }} \sim \bar{v} t_{\text {cor }} \ll l$, в следующих порядках по $n$ они становятся крупномасштабными, и поэтому ими нельзя пренебрегать на кинетических временны́х масштабах. Таким образом, начальные корреляции в членах высших (выше линейного) порядков разложения уравнения (54) по $n$ могут приводить к обрезанию обсуждаемых расходимостей в кинетическом уравнении при $t \sim t_{\text {rel }}$ [19]. Сказанное выше означает, что разложение "массового" (супер)оператора, определяющего эволюцию функции $f_{\mathrm{r}}(t)$ в уравнении (54), дает новую возможность разложения кинетических коэффициентов по $n$.

Уравнение (54) сводится к линейному однородному ОУУ со сверткой по времени, полученному в работе [15], если проекционный оператор $P$ и гамильтониан $H$ не зависят от времени и если выполнены соотношения (25) и (26).

Рассмотрим вопрос о существовании (сходимости) функции $R\left(t, t_{0}\right)$. Функция $R\left(t, t_{0}\right)$ хорошо ведет себя во все моменты времени. Более того, разложение ядра уравнения (54) может привести к сокращению полюсов функции $R\left(t, t_{0}\right)$. В работах $[15],[16]$ для линейного однородного ОУУ со сверткой по времени это было показано в линейном приближении по малой плотности для пространственно однородного разреженного газа классических и квантовых частиц. В этом случае не возникает проблемы с существованием функции $R\left(t, t_{0}\right)$. Глядя на выражение $(53)$, из которого следует соотношение $\left.\left(1-C_{0}\right)^{-1} C_{0}=f_{\mathrm{i}}\left(t_{0}\right) / f_{\mathrm{r}}\left(t_{0}\right)\right)$, можно ожидать, что то же самое имеет место для $R\left(t, t_{0}\right)(55)$ (см. ниже). 


\section{5. ЭВОЛЮЦИОННОЕ УРАВНЕНИЕ С УЧЕТОМ НАЧАЛЬНЫХ КОРРЕЛЯЦИЙ ДЛЯ РАЗРЕЖЕННОГО ГАЗА ЧАСТИЦ}

Применим уравнение (54) к рассмотренной в разделе 3 системе из $N \gg 1$ тождественных взаимодействующих классических частиц, описываемой оператором Лиувилля (31). Для получения уравнения для одночастичной функции распределения $F_{1}\left(x_{i}, t\right), i=1, \ldots, N$, мы будем использовать оператор $P(t)(34)$. Таким образом, учитывая (32), (40) и (41), получаем из (54) следующее уравнение для одночастичной функции распределения:

$$
\begin{aligned}
\frac{\partial F_{1}\left(x_{1}, t\right)}{\partial t}= & L_{1}^{0} F_{1}\left(x_{1}, t\right)+n \int d x_{2} L_{12}^{\prime} F_{1}\left(x_{2}, t\right) F_{1}\left(x_{1}, t\right)+D L^{\prime} C\left(t, t_{0}\right) f_{\mathrm{r}}(t)+ \\
& +D L^{\prime} \int_{t_{0}}^{t} d t^{\prime} R\left(t, t_{0}\right) \bar{U}\left(t, t^{\prime}\right)\left[\left(L^{0}-L_{1}^{0}\right)+Q\left(t^{\prime}\right) L^{\prime}-\frac{\partial P\left(t^{\prime}\right)}{\partial t^{\prime}}\right] f_{\mathrm{r}}\left(t^{\prime}\right) .
\end{aligned}
$$

Рассмотрим уравнение (56) в линейном приближении по параметру плотности (44). Поскольку в результате действия операторов $P(t)$ или $D$ получаются выражения, пропорциональные по крайней мере первой степени плотности $n$, а все члены в правой части уравнения (56) (кроме первого члена) также пропорциональны $n$ (или $D$ ), в уравнении (56) можно в первом приближении по $n$ пренебречь всеми членами с $P(t)$, включая таковые в выражении для $\bar{U}\left(t, t^{\prime}\right)$. Тогда в этом приближении оператор $\bar{U}\left(t, t^{\prime}\right)=U\left(t, t^{\prime}\right)$ и определяется выражением (45). Поэтому в (55) произведение $U^{-1}\left(t, t_{0}\right) \bar{U}\left(t, t_{0}\right)$ можно приближенно считать равным единице, а параметр $C_{0}\left(t, t_{0}\right)$ - приближенно равным $C_{0}$. Таким образом, как следует из (53) и (55), в принятом приближении параметр корреляции есть

$$
C\left(t, t_{0}\right)=U\left(t, t_{0}\right)\left[f_{\mathrm{i}}\left(t_{0}\right) f_{\mathrm{r}}^{-1}\left(t_{0}\right)\right] U^{-1}\left(t, t_{0}\right) .
$$

Для того чтобы вычислить вклад в эволюционное уравнение (56) начальных корреляций (57), следует использовать определения (37) и $(39)$ для функций $f_{\mathrm{r}}\left(t_{0}\right)$ и $f_{\mathrm{i}}\left(t_{0}\right)$.

Теперь, действуя, как в разделе 3, т.е. используя соотношения (32), (40), (46) и тот отмеченный выше факт, что каждое дополнительное интегрирование по фазовому пространству приводит к появлению дополнительной степени $n$, можно в первом приближении по $n$ представить уравнение (56) как

$$
\begin{aligned}
& \frac{\partial F_{1}\left(x_{1}, t\right)}{\partial t}=L_{1}^{0} F_{1}\left(x_{1}, t\right)+n \int d x_{2} L_{12}^{\prime}\left[1+C_{12}\left(t-t_{0}\right)\right] F_{1}\left(x_{2}, t\right) F_{1}\left(x_{1}, t\right)+ \\
& +n \int d x_{2} L_{12}^{\prime}\left[1+C_{12}\left(t-t_{0}\right)\right] \int_{0}^{t-t_{0}} d t_{1} e^{L_{12} t_{1}}\left[\left(L_{2}^{0}+L_{12}^{\prime}\right) F_{1}\left(x_{2}, t-t_{1}\right) F_{1}\left(x_{1}, t-t_{1}\right)+\right. \\
& \left.+\frac{\partial F_{1}\left(x_{2}, t-t_{1}\right)}{\partial t_{1}} F_{1}\left(x_{1}, t-t_{1}\right)\right]
\end{aligned}
$$

где

$$
\begin{gathered}
C_{12}\left(t-t_{0}\right)=e^{L_{12}\left(t-t_{0}\right)} \frac{g_{2}\left(x_{1}, x_{2}\right)}{F_{1}\left(x_{1}\right) F_{1}\left(x_{2}\right)} e^{-L_{12}\left(t-t_{0}\right)}, \\
L_{12}=L_{12}^{0}+L_{12}^{\prime}, \quad L_{12}^{0}=L_{1}^{0}+L_{2}^{0}, \quad F_{1}\left(x_{i}\right)=F_{1}\left(x_{i}, t_{0}\right) .
\end{gathered}
$$


Однородное нелинейное уравнение (58) является основным результатом данного раздела. Это новое уравнение, которое точно (в линейном приближении по $n$ ) описывает эволюцию одночастичной функции распределения неидеального классического газа частиц с произвольной пространственной неоднородностью и на любом временно́м масштабе. Это уравнение точно (в принятом приближении по $n$ ) учитывает начальные корреляции и описывает их единообразно со столкновениями между частицами и с другими процессами. Начальные корреляции входят в ядро памяти (массовый оператор), определяющее эволюцию одночастичной функции распределения, и не приводят к появлению нежелательных неоднородных членов в эволюционном уравнении (58) (ср. с (47)). Вклад начальных корреляций в процесс эволюции определяется зависящим от времени параметром корреляции (59), который описывает эволюцию по времени нормированной двухчастичной корреляционной функции $g_{2}\left(x_{1}, x_{2}\right) / F_{1}\left(x_{1}\right) F_{1}\left(x_{2}\right)$. Временна́я эволюция всех членов в уравнении (58) определяется точным двухчастичным пропагатором $G_{12}(t)=e^{L_{12} t}$.

Рассмотрим более подробно члены в правой части уравнения (58) и их эволюцию во времени. Первый член - это обычный член, определяемый потоком, обусловленным неоднородностью газа. Второй член представляет самосогласованное поле Власова, модифицированное начальными корреляциями. Если $C_{12}\left(t-t_{0}\right)$ обращается со временем в нуль (под действием оператора $\left.G_{12}\left(t-t_{0}\right)\right)$ при $t-t_{0} \gtrsim t_{\text {cor }}$ (см. ниже), мы получаем обычный власовский член. Третий член в правой части уравнения (58) описывает столкновения между частицами и влияние на них изменения одночастичной функции распределения на микроскопических пространственных и временны́х масштабах. Все эти процессы модифицированы начальными корреляциями, определяемыми корреляционной функцией $C_{12}\left(t-t_{0}\right)$. Таким образом, уравнение (58) учитывает влияние всех парных столкновений и корреляций на диссипативные и недиссипативные характеристики неидеального пространственно неоднородного газа частиц (что не имеет места в обычных подходах [19]).

Замечая, что член с $L_{2}^{0}$ имеет порядок $r_{0} / r_{h}$, а член с производной $F_{1}\left(x_{2}, t\right)$ по времени $-\sim t_{\text {cor }} / t_{\text {rel }}(\sim \gamma)$, мы можем пренебречь этими членами в принятом (линейном по $n$ ) приближении (если мы не интересуемся недиссипативными поправками к динамике неидеального газа). Тогда уравнение (58) принимает более простой вид

$$
\begin{aligned}
& \frac{\partial F_{1}\left(x_{1}, t\right)}{\partial t}=L_{1}^{0} F_{1}\left(x_{1}, t\right)+n \int d x_{2} L_{12}^{\prime}\left[1+C_{12}\left(t-t_{0}\right)\right] F_{1}\left(x_{2}, t\right) F_{1}\left(x_{1}, t\right)+ \\
& \quad+n \int d x_{2} L_{12}^{\prime}\left[1+C_{12}\left(t-t_{0}\right)\right] \int_{0}^{t-t_{0}} d t_{1} e^{L_{12} t_{1}} L_{12}^{\prime} F_{1}\left(x_{2}, t-t_{1}\right) F_{1}\left(x_{1}, t-t_{1}\right),
\end{aligned}
$$

где в последнем (столкновительном) члене функции распределения сталкивающихся частиц с принятой точностью $\sim r_{0} / r_{h} \ll 1$ могут быть взяты в одной и той же пространственной точке $\mathbf{r}_{1}=\mathbf{r}_{2}$ (в случае плазмы этого не следует делать во втором члене из-за дальнодействующего кулоновского взаимодействия).

Рассмотрим в качестве примера временну́ю эволюцию членов уравнения (60), обусловленных начальными корреляциями (членами с $\left.C_{12}\left(t-t_{0}\right)\right)$, когда зависимость 
начальной корреляционной функции и межчастичного взаимодействия от расстояния между частицами имеет вид

$$
g_{2}\left(x_{1}, x_{2}\right)=g_{0} e^{-r^{2} / r_{\text {cor }}^{2}} \phi\left(x_{1}, x_{2}\right), \quad V(r)=V_{0} e^{-r^{2} / r_{0}^{2}},
$$

где $\mathbf{r}=\mathbf{x}_{1}-\mathbf{x}_{2}-$ расстояние между частицами 1 и $2, r_{\text {cor }} \sim r_{0}, g_{0}$ и $V_{0}-$ постоянные параметры, а $\phi\left(x_{1}, x_{2}\right)$ - должным образом нормированная функция аргументов $x_{1}$ и $x_{2}$. Оценим временну́ю зависимость членов уравнения (60), обусловленных начальными корреляциями, в случае слабого межчастичного взаимодействия, когда временна́я эволюция определяется “свободным" пропагатором $G_{12}^{0}(t)=e^{L_{12}^{0} t}$. Тогда имеем (см. (49))

$$
G_{12}^{0}(t) \frac{g_{2}\left(x_{1}, x_{2}\right)}{F_{1}\left(x_{1}\right) F_{1}\left(x_{2}\right)}=g_{0} e^{-|\mathbf{r}-\mathbf{g} t|^{2} / r_{\text {cor }}^{2}} \frac{\phi\left(\mathbf{x}_{1}-\mathbf{v}_{1} t, \mathbf{p}_{1}, \mathbf{x}_{2}-\mathbf{v}_{2} t, \mathbf{p}_{2}\right)}{F_{1}\left(\mathbf{x}_{1}-\mathbf{v}_{1} t, \mathbf{p}_{1}\right) F_{1}\left(\mathbf{x}_{2}-\mathbf{v}_{2} t, \mathbf{p}_{2}\right)},
$$

где $\mathbf{g}=\mathbf{v}_{1}-\mathbf{v}_{2}-$ относительная скорость. Функция (62) стремится к нулю при $t \rightarrow \infty$ для любого фиксированного расстояния $\mathbf{r}$ и скорости $\mathbf{g}$. Используя (31), (61) и (62), можно представить член, определяющий вклад начальной корреляции во второй член в правой части уравнения (60), в виде

$$
\begin{aligned}
n \int d \mathbf{p}_{2} \int d \mathbf{r}\left[\frac{\partial}{\partial \mathbf{r}} V_{0} e^{-r^{2} / r_{0}^{2}}\right]\left(\frac{\partial}{\partial \mathbf{p}_{1}}-\frac{\partial}{\partial \mathbf{p}_{2}}\right) g_{0} e^{-|\mathbf{r}-\mathbf{g} t|^{2} / r_{\text {cor }}^{2}} \times \\
\quad \times \frac{\phi\left(\mathbf{x}_{1}-\mathbf{v}_{1} t, \mathbf{p}_{1}, \mathbf{x}_{1}-\mathbf{r}-\mathbf{v}_{2} t, \mathbf{p}_{2}\right)}{F_{1}\left(\mathbf{x}_{1}-\mathbf{v}_{1} t, \mathbf{p}_{1}\right) F_{1}\left(\mathbf{x}_{1}-\mathbf{r}-\mathbf{v}_{2} t, \mathbf{p}_{2}\right)} F_{1}\left(\mathbf{x}_{1}-\mathbf{r}, \mathbf{p}_{2}, t\right) F_{1}\left(\mathbf{x}_{1}, \mathbf{p}_{1}, t\right)
\end{aligned}
$$

(здесь для упрощения мы положили $\left.t_{0}=0\right)$. Легко видеть, что интеграл по $\mathbf{r}$ в $(63)$ не равен нулю, только если $t<t_{\text {соr }}$, где $t_{\text {cor }} \backsim r_{\text {cor }} / \bar{v} \sim r_{0} / \bar{v}$. При $t \gg t_{\text {сог он }}$ практически обращается в нуль в силу конечности радиуса межчастичного взаимодействия $r_{0}$. Физически это означает, что начальные корреляции распространяются наружу из рассматриваемой нами области, определяемой радиусом $r_{0}$, и в принятом линейном по $n$ приближении не возвращаются (см. также [18]). Конечно, такое поведение возможно, если вклад “параллельного движения" в члене (63) при малых $\mathbf{g}$ пренебрежимо мал. Таким образом, перемешивающий поток в фазовом пространстве необходим для гарантирования обращения в нуль членов, обусловленных начальными корреляциями. Такое же поведение в управляющем уравнении члена, обусловленного начальными корреляциями, было получено в работах [20], [21] в пределе ван Хова для квантово-механической системы, взаимодействующей с термостатом со свойством перемешивания. Член, обусловленный начальными корреляциями и дающий вклад в третий член в правой части уравнения (60) (интеграл столкновений), демонстрирует такое же поведение во времени. Таким образом, в рассмотренном примере обусловленные начальными корреляциями члены в уравнении (60) в принятом нами первом приближении по $n$ обращаются в нуль при $t \gg t_{\text {cor }}$.

Таким образом, если мы перейдем в уравнении (60) к кинетическому временно́му масштабу $t-t_{0} \gtrsim t_{\text {rel }} \gg t_{\text {cor }}$, то члены, обусловленные начальными корреляциями 
и определяемые двухчастичной корреляционной функцией $C_{12}\left(t-t_{0}\right)(59)$, обращаются в нуль на данном временно́м масштабе из-за перемешивающего потока в фазовом пространстве (как в рассмотренном выше примере), и поэтому уравнение (60) можно переписать в виде необратимого марковского кинетического уравнения:

$$
\begin{aligned}
\frac{\partial F_{1}\left(x_{1}, t\right)}{\partial t}= & L_{1}^{0} F_{1}\left(x_{1}, t\right)+n \int d x_{2} L_{12}^{\prime} F_{1}\left(x_{2}, t\right) F_{1}\left(x_{1}, t\right)+ \\
& +n \int d x_{2} L_{12}^{\prime} \int_{0}^{\infty} d t_{1} e^{L_{12} t_{1}} L_{12}^{\prime} F_{1}\left(x_{2}, t\right) F_{1}\left(x_{1}, t\right) .
\end{aligned}
$$

Для межчастичного взаимодействия с малым параметром $\varepsilon \ll 1$ вместо двухчастичного пропагатора $e^{L_{12} t}$ в столкновительном члене в уравнении (64) можно подставить “свободный" пропагатор $e^{L_{12}^{0} t}$ (см. (48)), тогда во втором приближении по $\varepsilon$ уравнение (64) сводится к уравнению Власова-Ландау (см., например, [18]). В пространственно однородном случае уравнение (64) совпадает с нелинейным уравнением Больцмана для функции распределения по импульсам $F_{1}\left(\mathbf{p}_{1}, t\right)$.

Заметим, что с помощью уравнения (58) можно в принятом приближении проследить все стадии эволюции рассматриваемой системы, начиная с начального обратимого режима $t_{0} \leqslant t \leqslant t_{\text {cor }}$. Если обратимые члены в уравнении $(58)$, связанные с начальными корреляциями $C_{12}\left(t-t_{0}\right)$, обращаются в нуль с течением времени, а последний интеграл в (64) существует, то эволюция системы может автоматически перейти из обратимого режима в необратимый, описываемый кинетическим уравнением (64).

\section{6. ЗАКЛЮЧЕНИЕ}

Выведены (а не постулированы) нелинейные ОУУ, описывающие эволюцию релевантной части функции распределения (статистического оператора). Новый подход, приводящий к этим уравнениям, основан на использовании нелинейного зависящего от времени оператора $P(t)$, определяющего релевантную часть функции распределения. Вообще говоря, этот оператор не является проекционным оператором. Полученное неоднородное нелинейное ОУУ (17) и (30) можно рассматривать как обобщение линейного ОУУ Накаджимы-Цванцига со сверткой по времени. Для случая не зависящих от времени проекционных операторов $P$ и $Q$ полученное уравнение редуцируется к обычному ОУУ со сверткой по времени. Неоднородное нелинейное ОУУ в равной мере полезно при выводе как нелинейных, так и линейных эволюционных уравнений для рассматриваемой редуцированной функции распределения, что отличает его от линейного ОУУ со сверткой по времени, которое, естественно, удобнее при выводе линейных эволюционных уравнений, например для подсистемы, взаимодействующей с термостатом. Используя полученное уравнение, мы вывели новое неоднородное нелинейное уравнение (47) для одночастичной функции распределения в линейном приближении по малому параметру - плотности газа классических частиц. В это уравнение, справедливое для произвольной 
пространственной неоднородности, входят пространственные и временнь́е изменения одночастичной функции распределения на микроскопических временнь́м масштабах (дающие вклад в недиссипативные характеристики неидеального газа и, таким образом, устраняющие несамосогласованность, имеющую место в стандартном подходе) и нерелевантная часть (источник), задаваемая начальной двухчастичной корреляционной функцией. Хотя полученное неоднородное нелинейное ОУУ позволяет вывести замкнутое нелинейное уравнение для одночастичной функции распределения, для получения кинетических уравнений Власова-Ландау или Больцмана по-прежнему требуются дополнительные допущения, такие как приближение случайных фаз или принцип Боголюбова ослабления начальных корреляций.

Для включения в рассмотрение начальных корреляций мы применили предложенный в работах [15], [16] метод для приведения неоднородного нелинейного ОУУ к однородному виду. Полученное точное однородное нелинейное ОУУ (54) для релевантной части функции распределения (статистического оператора) описывает все стадии эволюции рассматриваемой подсистемы, включая начальную стадию, когда играют роль начальные корреляции. Для вывода этого уравнения, которое можно использовать для получения как нелинейных, так и линейных эволюционных уравнений для редуцированных функций распределения, не надо использовать приближений типа приближения случайных фаз или принципа ослабления начальных корреляций. Начальные корреляции трактуются в этом уравнении единообразно со столкновениями посредством модифицированного ядра памяти, которое является отправной точкой для эффективного разложения по теории возмущений. Это уравнение было применено к пространственно неоднородному неидеальному газу классических частиц и в линейном по плотности приближении получено новое однородное нелинейное эволюционное уравнение (58) для одночастичной функции распределения, где начальные корреляции сохранены в функции памяти. Уравнение (58) описывает все стадии эволюции, учитывает все двухчастичные корреляции (столкновения) и преобразуется в нелинейное уравнение Больцмана или уравнение Власова-Ландау на соответствующем временно́м масштабе, когда все начальные корреляциии (и корреляции, обусловленные столкновениями) обращаются в нуль на данном временно́м масштабе. Таким образом, необратимые уравнения Больцмана и Власова-Ландау можно получить из уравнения Лиувилля без использования обычных дополнительных приближений, если динамика системы обладает необходимыми свойствами, такими как эргодический перемешивающий поток в фазовом пространстве.

\section{Список литературы}

[1] Н. Н. Боголюбов, Проблемы динамической теории в статистической физике, Гостехиздат, М.-Л., 1946.

[2] J. R. Dorfman, E. G. D. Cohen, J. Math. Phys., 8:2 (1967), 282-297.

[3] Дж. Ферцигер, Г. Капер, Математическая теория процессов переноса в газах, Мир, M., 1976.

[4] Ю. Л. Климонтович, Статистическая физика, Наука, М., 1982. 
[5] O. E. Lanford III, "Time evolution of large classical systems", Dynamical Systems, Theory and Applications, Lecture Notes in Phys., 38, ed. E. J. Moser, Springer, 1975, 1-111.

[6] D. Benedetto, F. Castella, R. Esposito, M. Pulvirenti, J. Stat. Phys., 116:1-4 (2004), 381-410.

[7] N. G. Van Kampen, J. Stat. Phys., 115:3-4 (2004), 1057-1072.

[8] S. Nakajima, Progr. Theoret. Phys., 20:6 (1958), 948-959.

[9] R. Zwanzig, J. Chem. Phys., 33:5 (1960), 1338-1341.

[10] И. Пригожин, Неравновесная статистическая механика, Синергетика: от прошлого к будущему, Едиториал УРСС, М., 2009.

[11] H.-P. Breuer, F. Petruccione, The Theory of Open Quantum Systems, Oxford Univ. Press, Oxford, 2002.

[12] B. Bellomo, G. Compagno, F. Petruccione, J. Phys. A, 38:47 (2005), 10203-10216.

[13] F. Shibata, Y. Takahashi, N. Hashitsume, J. Stat. Phys., 17:4 (1977), 171-187.

[14] F. Shibata, T. Arimitsu, J. Phys. Soc. Japan, 49:3 (1980), 891-897.

[15] V.F. Los, J. Phys. A, 34:33 (2001), 6389-6403.

[16] V.F. Los, J. Stat. Phys., 119:1-2 (2005), 241-271.

[17] Дж. Уленбек, Дж. Форд, Лекиии по статистической механике, Мир, М., 1965.

[18] Р. Балеску, Равновесная и неравновесная статистическая механика, Т. 2, Мир, М., 1978.

[19] Ю. Л. Климонтович, Кинетическая теория неидеалъного газа и неидеальной плазмы, Наука, М., 1975.

[20] S. Tasaki, K. Yuasa, P. Facchi, G. Kimura, H. Nakasato, I. Ohba, S. Pascazio, Ann. Phys., 322:3 (2007), 631-656.

[21] K. Yuasa, S. Tasaki, P. Facchi, G. Kimura, H. Nakasato, I. Ohba, S. Pascazio, Ann. Phys., 322:3 (2007), 657-676. 